\title{
RELATIONSHIP OF POST TRAUMATIC GROWTH PSYCHOLOGICAL DISTRESS AND QUALITY OF LIFE AMONG RENAL FALIURE PATIENTS
}

\author{
Neelam Ehsan, Nayab*, Adam Khan**, Amna Khan Shahid*** \\ International Islamic University, Islamabad Pakistan, *Shaheed Benazir Bhutto Women University, Peshawar Pakistan, **Fazaia Medical \\ College, Islamabad Pakistan, ${ }^{* * *}$ Islamic International Dental College, Islamabad Pakistan
}

\section{ABSTRACT}

Objective: To study the relationship and impact of post traumatic growth and psychological distress on quality of life among renal failure patients seeking dialysis, and to see differences in these variables among different age group patients.

Study Design: Cross sectional study.

Place and Duration of Study: Lady Reading Hospital, Peshawar, Government Hospital Mardan Pakistan, from Jan to Jun 2019.

Methodology: A sample of 300 renal failure Patients 18 years or older who had a diagnosis of end-stage renal disease receiving treatment within different dialysis units were approached through purposive sampling technique. Post traumatic growth inventory Quality of life scale and the Psychological distress scale were used to measure the study variables.

Results: Results reveal that psychological distress has a negative correlation with post traumatic growth ( $\mathrm{r}=-$ $\left.0.77^{* *}, p<0.01\right)$ and Quality of Life $\left(\mathrm{r}=-0.73^{* *}, p<.01\right)$. Whereas, Post Traumatic Growth has a strong positive correlation with Quality of Life $\left(\mathrm{r}=0.68^{*}, p<0.01\right)$. Similarly, Psychological distress $(\beta=-0.89, p<.000)$ and post traumatic growth $(\beta=0.25, p<.000)$ significantly predict quality of life and explained $57 \%$ variance $(\mathrm{F}(2,297)=198.4, p<0.000$, $\mathrm{R} 2=0.57)$ among the sample. Moreover, the results also show significant differences in the study variables between the two age groups (i.e., 16 to 32 years and 33 to 48 years) and it further suggest that middle age patients had higher levels of PTG as compared to younger and older patients.

Conclusion: This study reveals a significant negative relationship of post traumatic growth with psychological distress and a significant effect of post traumatic growth and psychological distress on the quality of life in the sample. This study also concludes that middle-age group patients had higher levels of post traumatic growth as compared to young and old patients.

Keywords: Dialysis, Renal failure patients, Posttraumatic growth, Psychological distress, Quality of life.

This is an Open Access article distributed under the terms of the Creative Commons Attribution License (http://creativecommons.org/licenses/by/4.0), which permits unrestricted use, distribution, and reproduction in any medium, provided the original work is properly cited.

\section{INTRODUCTION}

Kidneys are regarded as vital body organs necessary for a healthy life. Failure of kidney function in the human body is called end-stage renal disease (ESRD) which is a chronic medical condition for individual. Patients suffering from kidney failure face severe problems and survival risks due to the prolonged treatment of hemodialysis, however, survival chances increase if a kidney transplant is being carried out. In many cases, dialysis is an important kidney treatment and is required when patients own kidneys cannot perform their intended function. Generally,

Correspondence: Dr Neelam Ehsan, Department of Psychology, Islamic International University, Islamabad Pakistan

Received: 02 Dec 2020; revised received: 16 Dec 2020; accepted: 16 Dec 2020 the process of dialyses does not involve much pain, however, patients may suffer fear, stress, and other similar psychological states. Numerous studies on patients suffering from End-stage renal disease suggest psychological illnesses and symptoms by these patients ${ }^{1}$. These psychological conditions may involve depression, death-related anxiety, insomnia, fatigue, dementia, and various other psychological disorders.

A commonly observed negative psychological outcome of individuals with renal failure reported in the literature is psychological distress. It is an important psychological outcome of the individual undergoing the treatment of dialysis². Different mental health professionals describe psychological distress as a non-specific mental 
problem which comprises of combinations of symptoms including anxiety and depression ${ }^{3}$. The psychological distress in hemodialysis patients is a result of depression and anxiety that lead to lower social interaction with others and disrupted quality of life. For example, patients suffering from chronic diseases find their quality of life seriously impacted. In addition, from a medical perspective, chronic kidney disease is considered as a serious medical condition which has negative impact on the quality of life of individual patients 4 . In such conditions, patients require greater social support to cope with the pressure of physical and psychological challenges to overcome stress ${ }^{5}$. In this regard, Quality of life of patients is considered as an important concept and an indicator of a patient's overall wellbeing.

Moreover, patients undergoing dialysis mostly experience depressive affect ${ }^{6}$ which may also influence the prognosis of the medical treatment. It is also observed that patients having a higher level of depressive affect mostly stop complying with the nutritional restrictions advised by the physicians. Secondly, depressive affect is also associated with higher level of inflammatory process in the human body. As a result, patient is more vulnerable to vascular diseases and other medical complications. Depressive affect also negatively influences the therapeutic outcomes including higher mortality and bad prognosis ${ }^{7}$. Therefore, it is recommended that the patient's level of distress be evaluated continuously for making better treatment plan. The outcome of psychological distress is that it may cause patients to feel worthlessness. The distress among patients can be measured using indicators and based on the severity of such symptoms and their impact.

Studies have constantly stressed that patients suffering from serious illnesses are faced with multiple stressors regarding health and survival. Besides, they also added that these patients experienced some positive changes, as well in their lives in the form of Post traumatic growth. They further stressed that the reason for positive changes may be due to some indicators like achieving coping skills in patients who receive effective social support. Post-traumatic growth (PTG) refers to the positive psychological change which may emerge in the individual after passing through a traumatic event. Post Traumatic Growth (PTG) experience can facilitate an individual struggling with highly challenging life circumstances, especially in chronic disease conditions. Review of the literature suggests that event is likely to be traumatic if there is the death of a close relative, some significant loss, or becoming a victim of some life-threatening disease. This positive psychological change is a result that may emerge as he/she attempts to find new meaning and resolve due to the traumatic event. According to a recent study the considerable levels of post traumatic growth were observed in dialysisdependent patients ${ }^{8}$. The proponents of post traumatic growth stressed that it is different from optimism, emotional toughness or resilience since it not only involves the ability to cope and avoid damages from highly traumatic situation but includes the ability to develop growth which is beyond the pre-trauma level9. A comparison of people who experienced post traumatic growth will thus show greater adaptive responses as compared to what they had experience earlier before the traumatic situation arose.

One explanation of such growth is the human understanding that time cannot be reversed to undo undesirable things in life; however, life can be made more meaningful despite negative experiences ${ }^{10}$. The medical perspective on posttraumatic growth is based on the recognition that trauma can function as a spring board to an improved level of psychological functioning among individual.

\section{METHODOLOGY}

This study is designed to explore the relationship and effect of post traumatic growth and psychological distress on quality of life in renal failure patients. Similarly, we also aimed to find differences in the study variables within varying age groups based on social theories of aging in the present sample. Ethical approval was obtained from the Psychology Department Ethical 
committee, IIUI. The data was collected from Lady Reading Hospital Peshawar and Government hospital Mardan, Pakistan, from January to June 2019. Data was collected after getting the approval from the institutional authorities. Nonprobability convenience sampling technique was used to gather data from the sample. The inclusion criteria were adult patients who were about 18 years or older and had diagnosis of end stage renal disease and were currently receiving treatment or medical care from the nephrology departments within different dialysis units. They included all in patients, outpatients who were receiving dialysis who were ready to participate in the study. The participants were carefully approached and the purpose of the research was explained to them followed by getting their written informed consent for participation. Moreover, they were assured that the results of the study will be only utilized for the sake of research and their responses/identity will be kept confidential. The participants were provided with the study questionnaires along with the instructions and their queries were entertained. Each participant was attended individually and was thanked for his/her participation in the study. Exclusion criteria were participants with any type of documented mental illness or any type of physical disability.

To measure the study variables Psychological Distress Scale ${ }^{11}$ (K-10) having 10 items $(a=0.89)$ Quality of life Scale ${ }^{12}$ having 18 items $(\mathrm{a}=0.78)$ and a 21 item Post Traumatic Growth Inventory (PTGI)13 having alpha reliability of 0.71 were used. Keeping in view the education level of the sample Urdu versions of all the three scales attribute. The data was manually entered into the SPSS Statistics version 21. Frequencies and percentages for age, gender, educational qualification was calculated through descriptive statistics. The correlation method was used to see the correlation among study variables whereas ANOVA was run to see the differences between and among groups.

\section{RESULTS}

A total of 300 renal failure (male $=185$, female $=115$ ) patients age ranging from 18-81 years seeking dialysis from the aforementioned hospitals were approached to participate in the study. Only those patients who were willing to participate in the study were included.

Table-I shows that post traumatic growth negatively correlates with psychological distress $\left(\mathrm{r}=-.77^{* *}, p<0.01\right)$ however, it has positive correlation with Quality of life $\left(\mathrm{r}=.68^{*}, p<0.001\right)$. Similarly, Quality of Life is negatively correlated to psychological distress $\left(\mathrm{r}=-0.73^{* *}\right)$.

Table-I: Correlation matrix of study variables $(\mathrm{n}=300)$.

\begin{tabular}{l|c|c|c|c}
\hline Variables & $\mathbf{1}$ & $\mathbf{2}$ & $\mathbf{3}$ & $\mathbf{4}$ \\
\hline Age & - & .09 & 0.05 & 0.10 \\
\hline Quality of life & - & - & $-0.73^{* *}$ & $0.68^{* *}$ \\
\hline Psychological distress & - & - & - & $-0.77^{* *}$ \\
\hline $\begin{array}{l}\text { Post traumatic } \\
\text { growth }\end{array}$ & - & - & - & - \\
\hline$Q O L={ }^{*} p=<0.01$. & & - & -
\end{tabular}

Table-II show that Psychological distress $(\beta=-0.89, \quad p<0.000)$ and post traumatic growth $(\beta=0.25, p<0.000)$ significantly predict Quality of life and explain $57 \%$ variance $(\mathrm{F}(2,297)=198.4$, $p<0.000, \mathrm{R} 2=0.57$ ) among the sample.

Table-II: Prediction of quality of Life by psychological distress and post-traumatic growth $(n=300)$.

\begin{tabular}{l|c|c|c|c|c}
\hline Predictor Variable & $\begin{array}{c}\text { Unstandardized } \\
\text { Coefficient (B) }\end{array}$ & $\begin{array}{c}\text { 95\% Confidence } \\
\text { Interval }\end{array}$ & $p$-value & R2 & F \\
\hline Psychological Distress (K-10) & -0.89 & $-1.10--0.68$ & 0.000 & 0.57 & \multirow{2}{*}{$198.4^{* * *}$} \\
\hline Post-Traumatic Growth (PTGI) & 0.25 & $0.15-0.35$ & 0.000 & \\
\hline$* * *<0.01$
\end{tabular}

were used ${ }^{14}$. Higher scores on the scales represent high levels of Psychological Distress, Quality of Life and Post Traumatic Growth whereas lower scores represent lower levels of the respective

\section{DISCUSSION}

Kidney failure is considered as one of the medical emergencies and may be considered as a 
traumatic condition for the patient, therefore may likely impose psychological burden like psychological distress on the patient. On the other side, a bulk of previous researches also reported the development and existence of positive psychological development in the form of Post traumatic failures ${ }^{15}$. The positive growth in the form of PTG is an important yet relatively less understood concept. Barskova et al investigated the PTG and its association with health indicators among patients who were diagnosed with life-threatening diseases such as cancer, rheumatoid arthritis,

Table-III: Mean Standard deviation and F-value on Post traumatic growth, Quality of life and Psychological distress among dialysis dependent patients of different age groups $(n=300)$.

\begin{tabular}{|c|c|c|c|c|c|c|c|c|}
\hline \multirow{3}{*}{ Variables } & \multicolumn{8}{|c|}{ Age Groups } \\
\hline & \multicolumn{2}{|c|}{$\begin{array}{c}16-32 \\
(n=99)\end{array}$} & \multicolumn{2}{|c|}{$\begin{array}{c}33-48 \\
(n=114)\end{array}$} & \multicolumn{2}{|c|}{$\begin{array}{c}49-81 \\
(n=87)\end{array}$} & \multirow[t]{2}{*}{$\mathbf{F}$} & \multirow[t]{2}{*}{$\mathbf{P}$} \\
\hline & $\mathbf{M}$ & SD & $\mathbf{M}$ & SD & $\mathbf{M}$ & SD & & \\
\hline PTGI & 53.1 & 20.23 & 62.89 & 18.89 & 58.2 & 21.38 & 6.28 & $.002^{* *}$ \\
\hline QOLS & 59.85 & 17.93 & 68.20 & 17.93 & 63.40 & 16.45 & 6.33 & $.002^{* *}$ \\
\hline K-10 & 24.42 & 10.04 & 20.45 & 8.78 & 22.64 & 10.50 & 4.47 & $.012^{*}$ \\
\hline
\end{tabular}

growth among individuals who have faced traumatic situations. The present study therefore, aimed to explore the relationship and impact of Post traumatic growth (PTG) and psychological distress on quality of life among renal failure patients who were undergoing dialysis. Moreover, the difference in the levels of Post traumatic growth, Quality of life and Psychological distress among patients of different age groups, were also examined.

The present findings revealed a significant negative correlation of psychological distress with post traumatic growth and quality of life in the present sample. It's a natural phenomenon that distressed individual thinks negatively which may adversely influence the individual's quality of life therefore few chances to develop psychological growth exists. However, the important finding is that both growth and personal distress can co-exist in the individual after experiencing trauma. Calhoun et al suggests that while determining whether such growth occurred in an individual or not, one must compare the positive as well as negative changes in an individual due to the traumatic situation ${ }^{13}$. The review of literature also highlights the presence of moderate to high levels of post traumatic growth levels in individuals who are living with chronic illness such as stomach cancer survivors and individuals suffering from acquired brain injury and kidney multiple sclerosis, cardiac diseases, and HIV/ AIDS16. The findings of their study suggested that post traumatic growth is also associated with several factors including mental and physical health, coping strategy employed by an individual, availability of social support, quality of relationships and so on.

The present study also revealed a significant positive association of PTG with quality of life among the sample. The relationship between post-traumatic growth and quality of life is logical for an individual who experience post-traumatic growth will appreciate life and can experience a better quality of life. Literature review suggest that quality of life of such patients is generally impacted due to the negative outcomes of the disease. Furthermore, factors which contribute to the quality of life among patients having a critical illness or related treatments leads to disability or pain, and thus can reduce the quality of life. Past literature establishes that treatment and disease-related factors contribute into the reduced quality of life among patients having end-stage renal disease. According to the National Service Framework, patients having such disease necessarily require psychological assistance to cope with newer life style and associated challenges (Department of Health, 2005. The relationship is also supported by a bulk of previous studies. For example; Kimmel observed a positive 
relationship between post-traumatic growth and quality of life through an empirical study ${ }^{17}$. Similarly, Gerogianni et al also found a positive association between post-traumatic growth and quality of life ${ }^{18}$. Several other studies also found positive association between post traumatic growth and quality of life of patients or individuals suffered from some major illness or trauma19,20.

The present study further aimed to investigate differences in post-traumatic growth, psychological distress and quality of life in young, middle age and older patients. The findings revealed that middle-age patients scored high on Post traumatic growt has compared to young and older patients. Moreover, young age group patients were comparatively higher on psychological distress as compared to the middle age and older patients. The possible reason for the significant difference among the three groups on PTG and psychological distress might be attributed to the high levels of the burden that this disease imposes on the young and old patients. All groups were experiencing the same physical and psychological complaints and were equally suffered by the illness. However, the middle age groups (33 to 48 years) may be considered the most mature age group in terms of their age and coping abilities as compared to younger and older age group. They are probably better able to find new meaning to their physical state and develop positive change in coping with the disease condition as compared to young and old age counterparts. While on the other hand, young age individuals may become easily distressed by this kind of trauma. They are not mature enough to think positively or cope with such disease condition. This group of patients might also have a poor concept of adjustment because of their age, and are not much strong and mature like their middle age counterparts. They are generally careless about their health condition which affects psychological health as well as their quality of life. Such findings suggest that this group because of their deteriorated physical condition may have trouble in maintaining a better quality of in the aftermath of a chronic illness condition. On the contrary, however, past research findings suggest that while dealing with chronic illnesses the post traumatic growth did not reveal many differences in terms of age groups e.g., a study conducted did not find a statistical difference in post-traumatic growth levels among different age groups having chronic medical conditions ${ }^{21}$. Similarly, Post traumatic growth between youth and adults observed is quite similar among cancer patients and its existence among youth is a conceptual psychological idea however it is quite complex and is still considered as primarily hypothetical 22,23 .

\section{CONCLUSION}

PTG experience can facilitate individuals struggling with highly challenging life circumstances especially if they are suffering from any health condition. Little is known about its relationship with psychological distress and several other demographic variables among patients undergoing dialysis. This study reveals a significant negative relationship of post traumatic growth with psychological distress and positive relationship with quality of life among the said group. This study further concludes that theage of patients having renal failure may be considered as theprimary factor influencing PTG. We found that middle age group patients were significantly higher on PTG, however younger and older age patients were higher on psychological distress. As literature suggests that PTG can be useful in patients who are struggling with disease conditions. Therefore, health care providers and caregivers should pay special attention to young and old patients to reduce psychological distress and promoting PTG to help them in the recovery process.

\section{CONFLICT OF INTEREST}

This study has no conflict of interest to be declared by any author.

\section{REFERENCES}

1. Bayat A, Kazemi R, Toghiani A, Mohebi B, Tabatabaee MN, Adibi N. Psychological evaluation in hemodialysis patients. J Pak Med Assoc 2012; 62 (3 Suppl-2): S1-S5.

2. Cos TA. Stress coping, and psychological distress: An examination into the experience of individuals utilizing dialysis for 
end-stage renal disease. Dissertation submitted to the faculty of Drexel University. 2008.

3. Drapeau A, Marchand A, Beaulieu-Prévost D. Epidemiology of psychological distress. Mental illnesses-understanding, prediction and control 2012; 69(1): 105-06.

4. Kimmel PL, Peterson RA, Weihs KL, Simmens SJ, Alleyne S, Cruz I, et al. Multiple measurements of depression predict mortality in a longitudinal study of chronic hemodialysis outpatients. Kidney Int 2000; 57(5): 2093-98.

5. Taylor S. Anxiety sensitivity: Theory, research, and treatment of the fear of anxiety. Routledge 2014 May 12; 1-384. Available from: https://www.routledge.com/Anxiety-Sensitivity-theoryResearch-and-Treatment-of-the-Fear-of-nxiety/Taylor/p/book/ 9781138012479

6. Kimmel PL. Psychosocial factors in dialysis patients. Kidney Int 2001; 59(4): 1599-613.

7. Tsay SL, Healstead M. Self-care self-efficacy, depression, and quality of life among patients receiving hemodialysis in Taiwan. Int J Nurs 2002; 39(3): 245-51.

8. Yorulmaz H, Bayraktar S, Özdilli K. Post traumatic growth in chronic kidney failure disease. Procedia-Soc Behav Sci 2010; 5(1): 2313-19.

9. Tedeschi RG, Calhoun LG. Posttraumatic growth: Conceptual foundations and empirical evidence. Psychol Inq 2004; 15(1): 1-8.

10. Joseph S. Client centred therapy, post traumatic stress disorder and post traumatic growth: Theoretical perspectives and practical implications. Psychol Psychother-T 2004; 77(1): 101-19.

11. Kessler R, Mroczek D. Final versions of our non-specific psychological distress scale. Memo dated March 1994; 10: 1994.

12. Flanagan JC. Measurement of quality of life: current state of the art. Arch. Phys Med Rehabil 1982; 63(2): 56.

13. Calhoun LG, Tedeschi RG. Facilitating posttraumatic growth: A clinician's guide. Routledge; 1999.

14. Yousaf M, Kauser R. Self Care: Coping strategies and quality of life of individuals with diabetes. J Behav Sci 2016; 26(1): 17-25.

15. Sim BY, Lee YW, Kim H, Kim SH. Post-traumatic growth in stomach cancer survivors: Prevalence, correlates and relationship with health-related quality of life. Eur J Oncol Nurs 2015; 19(3): 230-36.

16. Barskova T, Oesterreich R. Post-traumatic growth in people living with a serious medical condition and its relations to physical and mental health: A systematic review. Disabil Rehabil 2009; 31(21): 1709-33.

17. Kimmel PL, Peterson RA. Depression in end-stage renal disease patients treated with hemodialysis: tools, correlates, outcomes, and needs. Sem Dial 2005; 18(2): 91-97.

18. Gerogianni SK, Babatsikou FP. Psychological aspects in chronic renal failure. Health Sci J 2014; 8(2): 205.

19. Yucetin L. Investigation of post traumatic growth among living kidney donors. The $5^{\text {th }}$ Annual meeting of global nephrologists; 2016.

20. Yorulmaz H, Bayraktar S, Özdilli K. Posttraumatic growth in chronic kidney failure disease. Procedia-Soc Behav Sci 2010; 5(1): 2313-19.

21. Cukor D, Cohen SD, Peterson RA, Kimmel PL. Psychosocial aspects of chronic disease: ESRD as a paradigmatic illness. J Am Soc Nephrol 2007; 18: 3042-55.

22. Kilmer RP, Gil-Rivas V, Griese B, Hardy SJ, Hafstad GS, Alisic E. Post traumatic growth in children and youth: clinical implications of an emerging research literature. Am J Orthopsychiatry 2014; 84(5): 506-18.

23. Glad KA, Jensen TK, Holt T, Ormhaug SM. Exploring selfperceived growth in a clinical sample of severely traumatized youth. Child Abuse Negl 2013; 37(5): 331-34. 